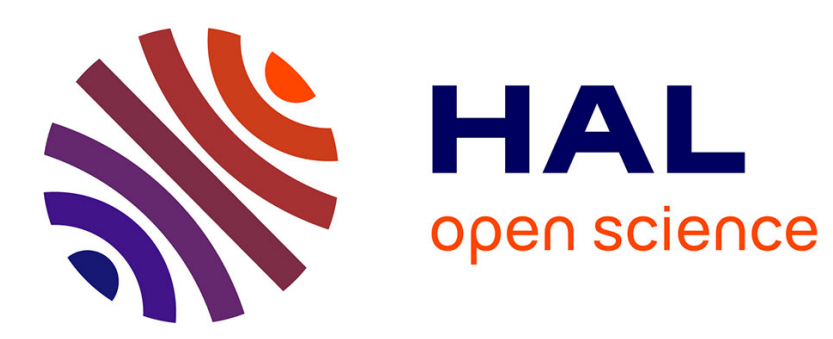

\title{
A propos d'un domaine linguistique normé mais peu coté: l'orthographe à l'école
}

\author{
Danièle Manesse
}

\section{To cite this version:}

Danièle Manesse. A propos d'un domaine linguistique normé mais peu coté: l'orthographe à l'école. Langage et Société, 2007, Les normes pratiques, 119, pp.81-92. 10.3917/ls.119.0081 . hal-01435859

\section{HAL Id: hal-01435859}

\section{https://hal-univ-paris3.archives-ouvertes.fr/hal-01435859}

Submitted on 15 Jan 2017

HAL is a multi-disciplinary open access archive for the deposit and dissemination of scientific research documents, whether they are published or not. The documents may come from teaching and research institutions in France or abroad, or from public or private research centers.
L'archive ouverte pluridisciplinaire HAL, est destinée au dépôt et à la diffusion de documents scientifiques de niveau recherche, publiés ou non, émanant des établissements d'enseignement et de recherche français ou étrangers, des laboratoires publics ou privés. 


\section{À propos d'un domaine linguistique normé mais peu coté: l'orthographe à l'école}

\section{Danièle Manesse}

Université Paris III

daniele.manesse@univ-paris3.fr

L'orthographe française est, pour une part, retorse et byzantine. On sait comment s'est construit sur cinq siècles le système brouillé de la transcription graphique du français, et de quels facteurs résulte sa grande complexité (Chervel 1969) ${ }^{1}$. Des travaux récents ont montré que l'orthographe, en évolution constante depuis le XVI a cessé d'évoluer vers 1835, après que la loi Guizot en a eu rendu l'enseignement obligatoire (Chervel 2006).

La complexité de l'orthographe a deux effets contraires. D'une part, elle conforte son statut de savoir populaire car sa complexité en naturalise les difficultés: comment mettre en cause un système aux règles souvent impénétrables, hérité, que tout le monde a appris à grands frais, à l'école obligatoire dès la jeune enfance? Dans le même temps, elle suscite l'indifférence des spécialistes du langage, qui la renvoient, non sans quelque raison, du seul côté de la norme, manipulée par des interventions autoritaires, celles de l'Académie française, de l'école et de grammairiens, lesquelles ont contribué à la constituer en prescription arbitraire avec tous les effets qu'elle induit (fonction de sélection et d'exclusion, marque de distinction, etc.).

Or les sciences du langage ont à mon sens, joué - par principe à bon droit - un rôle décisif dans les choix qui ont présidé aux évolutions

1. C. Blanche-Benveniste \& A. Chervel 1969; V.G. Gak 1976; N. Catach, 1978. 
importantes de la manière d'enseigner le français à l'école depuis vingt ans: les programmes scolaires, notamment ceux du collège, organisés depuis 1996 autour de la "maîtrise² du discours ", témoignent de leur influence, tant dans les contenus que dans les méthodes; pour dire vite, en lieu et place des enseignements normés traditionnels de la langue, les pratiques en classe s'appuient sur des pratiques descriptives centrées sur la tolérance à la variation et l'évitement des modes d'enseignements dogmatiques, règles et mémorisation.

\section{Deux évaluations}

En 1986-1987, André Chervel et moi-même avons mené une enquête sur le niveau orthographique des élèves de 10 à 16 ans, comparé à un siècle de distance, à partir de la découverte aux Archives nationales par A. Chervel d'un gisement très important de copies d'élèves de la fin du XIX', dispersé et jusqu'alors inexploité: des dictées d'un même texte, qu'on a désigné par l'incipit les arbres. Nous avions alors circonscrit, puis exploité ce corpus comme un témoignage global du niveau en orthographe des élèves de l'époque, et organisé une comparaison avec les élèves de mêmes âges en 1987. Ce travail s'inscrivait dans le débat qui agitait (déjà) l'opinion sur la baisse supposée du niveau des élèves, en ces années de bouleversement consécutives à la mise en place du collège unique. Notre objectif était simple: nourrir ce débat avec des éléments de preuve "scientifique ». Les résultats de la comparaison des deux populations d'élèves, fondée sur des analyses solides, étaient en faveur des élèves de 1987, et nous ont conduits à un bilan satisfaisant d'un siècle d'école « républicaine».

Ce choix de l'orthographe pour se placer dans la polémique sur le niveau des élèves pouvait paraître étrange, de la part de deux chercheurs linguistes l'un et l'autre; de par la nature de leur engagement disciplinaire, on pouvait s'attendre à ce qu'ils soient réticents à ancrer leur travail dans un domaine si discuté: le système orthographique du français. De fait, A. Chervel et moi pensions et pensons encore que l'état de l'orthographe française pèse de manière

2. D. Manesse applique les rectifications orthographiques de 1990. 
insupportable sur l'école, et sur les sociétés de langue française en général. L'un et l'autre, nous sommes partisans de simplifications importantes de l'orthographe du français. Mais nous n'avions pas le choix: aucun autre exercice scolaire n'était resté stable sur un siècle et la dictée dite Les arbres a fourni le premier legs du passé de travaux d'élèves réels utilisables pour effectuer une mesure qui échappe aux fantasmes nostalgiques.

Les critiques qu'on a pu faire sur le caractère démesuré de l'intérêt attribué à l'orthographe dans la tradition scolaire ne doivent pas masquer son rôle dans les activités de construction de la langue écrite: telle qu'elle est, l'orthographe constitue un révélateur et un outil des acquisitions scolaires, de points de vue multiples. L'acquisition de l'orthographe est une des voies qui impose de penser la distance entre la langue orale et la langue écrite; dans l'orthographe se mesure la capacité à respecter des normes, celles évidemment du système de l'écrit, mais bien aussi une norme symbolique profondément valorisée dans le corps social. Et, quelles que soient les critiques qu'on puisse porter sur sa complexité inutile eu égard à la fonction qu'elle doit assurer, l'orthographe oblige à mettre en œuvre de la part des élèves qui l'apprennent à l'école des processus réflexifs, de nature métalinguistique, du type de ceux qui sont à l'œuvre dans la plupart des apprentissages scolaires En tant que telles, les activités orthographiques à l'école, bien au-delà de l'acquisition du savoir orthographique, constituent un des aspects du rapport à la langue écrite, versant complémentaire de la lecture dans l'ensemble de compétences nommé souvent littératie (voir la dernière livraison en 2007 de la revue Le Français Aujourd'hui).

Sans état d'âme, j'ai donc pensé utile, quasiment vingt ans après, de renouveler l'évaluation Chervel-Manesse de l'orthographe des adolescents scolarisés dans l'école obligatoire, en 2005, à l'identique de celle de 1987, pour permettre la comparaison ${ }^{3}$. Cette décision est née des réflexions que $\mathrm{m}^{\prime}$ avait inspirées un travail précédent, mené en compagnie d'une équipe de jeunes professeurs

3. Danièle Cogis, Michèle Dorgans et Christine Tallet et moi-même. J'écris le présent texte dans le temps même de la rédaction de l'ouvrage L'orthographe: à qui la faute? (février 2007). Il est par force une sorte de condensé de certains des points qui y sont traités. 
dans les «classes difficiles» de ZEP (Manesse 2003). Ce travail cherchait à comprendre pourquoi, du point de vue des résultats scolaires à l'évaluation lors de l'entrée en sixième, les élèves des collèges de ZEP obtenaient, en moyenne, des résultats moyens très inférieurs à ceux des élèves de l'ensemble des collèges; pis encore, pourquoi, malgré tous les moyens investis dans leur scolarisation, une dynamique de marginalisation croissante de ces élèves par rapport à la réussite scolaire semblait être en marche, comme le montraient nombre d'études. Au cœur des résultats de cette recherche, on pouvait identifier la grande insécurité des élèves de ZEP en matière de lecture-écriture, source de souffrance pour eux et de découragement pour leurs professeurs; par ce travail, je pense avoir contribué à montrer que la mise en œuvre (et non l'intention) des nouveaux programmes de français au collège, centrés sur la maîtrise du discours ${ }^{4}$ avait une responsabilité dans les difficultés linguistiques des élèves de ZEP. Cette nouvelle recherche sur l'orthographe s'est donc inscrite dans la suite de celle de 2003, et elle visait à évaluer où en était toute la population de l'école obligatoire dans sa compétence orthographique.

La nouvelle enquête s'est donc déroulée dans 25 collèges du territoire métropolitain, appartenant à échantillon de collèges public tiré par la DEP, et dans 25 écoles associées à ces collèges; $20 \%$ de l'ensemble de ces établissements étant classés ZEP. L'objectif de cette recherche est double: mesurer l'évolution des compétences en quasiment vingt ans et tenter d'en comprendre les ressorts.

Nous avons choisi deux approches dans le corpus des 37000 «formes erronées » (sur un total de 170000 mots) que nous avons saisies en machine: une approche quantitative permettant de comparer les résultats de la génération 1987 et ceux de la génération 2005; une analyse qualitative fondée sur le classement des erreurs en huit types différents, relevant de trois grands domaines de la mise à l'écrit: les erreurs de langue (mots non segmentés, non identifiés etc.), de grammaire (accord et conjugaison) et de lexique (formes non normées dites d'orthographe d'usage, accents).

4. Appliqués en 1996 en sixième, 1997 en cinquième, etc. 


\section{Des résultats en baisse sensible entre 1987 et 2005}

Il est incontestable en premier lieu que nous sommes en présence d'un phénomène massif: en une génération, l'orthographe des élèves s'est détériorée. Il est aussi certain que ces résultats, touchant un domaine surinvesti de passion et contigu à celui de la lecture, laquelle fait actuellement l'objet d'un débat chaotique, méritent une présentation à la fois prudente et argumentée, que nous nous efforçons de faire, mais qu'on ne peut développer dans le cadre de ce court article. Qu'on pardonne le caractère un peu abrupt des données présentées par la suite puisqu'on peut se reporter à l'étude entière pour satisfaire des exigences légitimes de rigueur.

Il s'agira ici évidemment de moyennes. Les "scores » qu'ont obtenus les élèves sont proportionnels au nombre d'erreurs dans leur dictée: un score de 24 points correspond à 12 erreurs de deux points, le taux de tous les types d'erreurs, à l'exception des erreurs de « signes orthographiques » (accents, apostrophe et trait d'union qui ne "pèsent» qu'un point). Ces scores sont beaucoup plus lourds en 2005 qu'ils ne l'étaient en 1987. Le score moyen en 2005 est de 27 points, soit 13,5 fautes « lourdes »; il était de 16 points en 1987, soit huit fautes: les élèves d'aujourd'hui font donc beaucoup plus d'erreurs qu'il y a 20 ans, et ceci à chaque niveau de la scolarité. L'écart entre les résultats des élèves de 1987 et ceux de 2005 est en moyenne de deux niveaux scolaires: les élèves de cinquième de 2005 font le même nombre de fautes que les élèves de CM2 il y a vingt ans; les élèves de troisième de 2005, le même nombre d'erreurs que les élèves de cinquième de 1987, etc. On doit prendre garde que cet écart concerne cependant des élèves d'âge différent: le décalage entre les performances des deux générations est de deux classes, mais il n'est que d'un an et demi d'âge, car les élèves sont en moyenne plus jeunes de six mois: ils sont moins « orientés » dans des filières marginales, et redoublent les classes en moins grand nombre qu'il y a vingt ans. 
SCORES COMPARÉS PAR CLASSE (1 987-2005)

Scores ramenés au demi-point

\begin{tabular}{|l|l|l|l|l|l|l|}
\hline & CM2 & $6^{\mathrm{e}}$ & $5^{\mathrm{e}}$ & $4^{\mathrm{e}}$ & $3^{\mathrm{e}}$ & ensemble \\
\hline 1987 & 24,5 & 21 & 16,5 & 10,5 & 8 & 16 \\
\hline 2005 & 36 & 31,5 & 27 & 23 & 17,5 & 27 \\
\hline
\end{tabular}

Les élèves de 2005, comme leurs aînés de 1987, progressent en moyenne à chaque niveau scolaire; mais compte tenu de la grande différence des scores de départ en CM2, entre 1987 et 2005, la progression est bien moins rapide qu'il y a 20 ans: entre le CM2 et la troisième les élèves de 1987 divisaient par trois leur nombre d'erreurs, ils ne le divisent plus que par deux en 2005. On pouvait $s^{\prime}$ attendre à de moindres performances chez ceux des élèves scolarisés en ZEP: l'écart des moyennes de chaque classe est en gros d'une année scolaire entre le système ZEP et les élèves hors-ZEP dans nos résultats de 2005.

Pour ce qui concerne la nature des erreurs commises par les élèves, il apparaît d'abord qu'entre 1987 et 2005, les comportements orthographiques n'ont pas substantiellement changé: la hiérarchie dans la répartition des fautes selon les divers types reste grosso modo la même. Mais un résultat notable saute aux yeux: si en 2005 les erreurs grammaticales pèsent le plus lourd, comme c'était le cas en 1987, leur poids dans l'ensemble des erreurs a fait un bond important entre les deux enquêtes et ce sont elles, beaucoup plus nombreuses dès le CM2, qui plombent les résultats de 2005. Ces erreurs diminuent certes de classe en classe, en 2005 comme en 1987. Mais, tandis que les élèves d'il y a vingt ans divisaient par quatre le taux d'erreurs grammaticales entre le CM2 et la troisième, ceux de 2005 les divisent à peine par deux. Les élèves de ZEP partent avec un handicap lourd de ce point de vue; mais entre le CM2 et la cinquième, eux aussi progressent laborieusement comme les autres en orthographe grammaticale (même s'ils ne rattrapent pas l'année de retard avec les élèves hors ZEP). Cette progression en ZEP comme hors ZEP contribue ainsi à une homogénéité des 
comportements orthographiques des élèves, quel que soit le réseau où ils étudient: les difficultés sont les mêmes pour tous.

On doit considérer de manière différente l'écart entre les résultats des élèves de 1987 et ceux de 2005 selon le niveau scolaire qu'on envisage. On peut penser que l'école primaire s'est déchargée d'une partie de l'enseignement de l'orthographe sur les quatre années d'école obligatoire du collège, ce qu'attestent les directives en vigueur. Mais le recul des élèves de troisième mérite plus d'attention, sinon de préoccupation, pour la raison qu'ils n'auront plus, dans leur scolarité ultérieure, d'enseignement de langue.

\section{Comprendre la régression}

\section{À l'école}

Une première donnée: le temps accordé à l'enseignement du français dans l'école s'est certainement réduit en vingt ans, comme le confirme l'étude des programmes et prescriptions. Il ne suffit pas cependant de considérer le temps imparti à la discipline: il importe aussi de comprendre, dans les grandes réorientations des plans d'étude, comment la nature du travail sur l'orthographe s'est modifiée.

Depuis 1995, les textes de l'enseignement primaire déconseillent les apprentissages systématiques, la mémorisation, les exercices répétitifs. L'allégement progressif de la liste des notions de grammaire et d'orthographe au fil des programmes trouve son explication dans cette volonté explicite: tout le poids des apprentissages formels ne doit pas reposer pas sur le temps de l'école primaire, et il revient au collège d'assurer une part de leur enseignement. Cependant, cette attente est déçue: en effet, dans le même temps depuis 1996, les programmes du collège ont été aspirés "vers le haut », privilégiant les activités métatextuelles en organisant le curriculum, comme susdit, autour de la maîtrise du discours. La philosophie qui inspire les programmes du collège est d'ancrer les activités de langue dans la confrontation avec des textes réels: comme à l'école primaire, le travail décontextualisé sur la langue, souvent jugé "mécanique», est proscrit, au profit d'activités " décrochées » activées à l'occasion de l'étude ou la production d'un texte au cours de la séquence. L'entrée dans les programmes 
du collège de nouveaux savoirs, notamment autour des théories de l'énonciation, a secondarisé l'étude des « outils de la langue»: grammaire, lexique, et celle de l'orthographe en particulier. Les professeurs de collège vivent ainsi dans une tension, pour l'heure irrésolue, entre la conception ancienne et discréditée de l'étude de la langue "pour elle-même ", et celle, nouvelle et auréolée de la caution scientifique, de l'entrée dans la langue par les textes.

L'orthographe, dans les vagues des réorganisations curriculaires, est ainsi une sorte de patate chaude: l'école compte sur le collège pour la stabiliser, et le collège, centré sur la maîtrise du discours, la suppose en gros acquise dans ses grandes lignes quand les élèves entrent en sixième. Le mouvement ne s'arrête pas là! Déplaçons-nous au niveau de l'université: les professeurs de l'enseignement supérieur s'agacent des déficiences orthographiques de leurs étudiants, mais ils ne jugent pas de leur ressort de l'enseigner:

Ce n'est pas une des moindres contradictions de l'enseignement universitaire actuel que de déplorer la maîtrise incertaine de l'orthographe chez les étudiants, jusque dans les concours d'enseignement, sans pour autant mettre en place une étude raisonnée du système graphique de français (Elalouf et al. 1998: 5).

L'institution témoigne du désarroi des professeurs de collège en matière d'enseignement de l'orthographe. Ils sont à la fois submergés par le nombre d'erreurs que commettent les élèves, et soucieux d'inscrire leur travail dans le cadre des nouveaux programmes, centrés sur la maîtrise du discours et organisés en séquences, au point de poser « fréquemment » cette question: «A-t-on le droit de consacrer des séances (de grands moments) à l'orthographe $?^{5}$ ».

De fait, les professeurs doivent placer leur travail entre deux pôles contraires: à la fois ils sont partie prenante de l'opinion publique, celle qui s'émeut sitôt qu'un indice, un événement, font supposer que la «langue est menacée »; mais dans l'école, ils suivent massivement la nouvelle dynamique qui leur est proposée. Bernadette Wynants (1997), citée ici même par Nicole Ramognino,

5. Inspection générale, Rapport à monsieur le ministre «L'enseignement du français en collège », 2002. « Droit » mis en italique pas DM. 
témoigne du caractère problématique qu'a pris pour eux l'enseignement de l'orthographe, la forme en quelque sorte la plus «voyante » et la plus populaire de la norme linguistique, qu'on leur demande maintenant de relativiser au profit d'enjeux supérieurs.

Plus largement, l'orthographe est-elle une norme en voie de relégation?

Le système orthographique du français, très complexe, pour être transmis dans l'école à des générations d'élèves avec des résultats qui $n^{\prime}$ ont jamais été parfaits, a exigé beaucoup de temps, d'exercices répétés, de règles ressassées, de dictées agressives, d'ingéniosité mnémotechnique. L'école a pu le faire parce qu'elle s'appuyait sur une valeur qu'elle a contribué à promouvoir dans le peuple et qui faisait consensus, même de ceux pour qui elle générait de l'oppression: la langue écrite et ses exigences formelles.

Crise des valeurs partagées, éclatement des normes sociales... Ces questions qui traversent ce dossier, pourraient bien concerner l'orthographe de la langue écrite, partie la plus formelle et la plus visible de l'objet d'enseignement « langue ». En effet, la réorientation à l'école sur les pratiques de discours, de lecture, de production d'écrit et le développement en classe de la confrontation vivante dans le débat et l'argumentation, induits par les efforts conjugués des chercheurs novateurs en didactique de la langue et de l'Institution ne se sont pas encore accompagnés d'une réflexion sur la manière d'articuler les espaces ouverts des textes avec le caractère fermé et rigide des activités normatives, au premier chef l'orthographe. Si tel était le cas, on pourrait considérer que le recul orthographique de la génération actuelle d'élèves serait un reflet de cet impensé. Élargissons encore le propos: la langue «nationale » est une valeur traditionnellement respectée: la langue unit, fait référence et suscite la révérence. Les temps ne sont-ils pas en train de changer sous nos yeux, sans qu'on puisse prendre la mesure de ces changements, parce que nous sommes pris dans ce mouvement? Dans sa réflexion sur la discipline à l'école - et l'orthographe est bien une forme de discipline de la langue - Eirick Prairat évoque ce temps où nous sommes: 
Les attitudes scolaires et les normes comportementales ne font plus l'objet d'un consensus. D'une manière générale, on peut dire que l'exigible en matière de discipline scolaire est devenu flou et mal défini [...] L'émiettement des repères, l'affaiblissement du sens de l'interdit ou encore la perte des effets structurants de l'obligation ne sont que des déclinaisons de cette crise de la fonction symbolique qui est crise du lien, défaut d'articulation, effritement généralisé de tout ce qui nous relie et nous articule (2002: 20 et 40).

Hors l'école. Une autre conception de l'écrit dans la société?

De nouvelles formes d'écrit sont apparues, rapidement popularisées, notamment dans les pays nantis; ainsi en est-il de l'écrit des courriers électroniques, qui n'a pas en général pas cette caractéristique fondamentale de la langue écrite, sa vocation à durer: comme l'oral, les méls sont destinés à disparaître sans trace. Il est prématuré d'imputer l'attention moindre portée à l'orthographe dans l'école aux formes de rédaction de ces nouvelles formes d'écrit, méls et textos, comme certains le font: certaines recherches en font douter ${ }^{6}$; on peut rappeler d'ailleurs que les scripteurs, y compris les plus experts d'entre eux, ont toujours utilisé des codes très peu normés, adaptés aux circonstances de leur production: la prise de note en est un exemple répandu, pratiqué à l'école, sans que nul y ait eu à redire. Les adolescents ne se méprennent d'ailleurs pas sur les normes de la langue scolaire: dans le corpus considérable de dictées d'élèves dont nous disposons, plus de 170000 mots, seules deux occurrences rappellent la pratique du texto, maintenant quotidienne pour nombre d'entre eux: par deux fois, « de » est écrit « 2 ».

Il est bien trop tôt pour conclure à quoi que ce soit sur le sujet, quand ces techniques ne se sont répandues que depuis quelques années. Cependant, les nouvelles pratiques d'écrit diffusent dans la vie quotidienne un écrit réduit à n'être qu'un simple canal de communication, soumis à aucun contrôle, et par là, elles contribueraient peut-être à construire une représentation de la langue écrite dépouillée de contraintes, à rebours de la conception complexe que l'école cherche à construire. Les écrits virtuels participeraient peut-être à nourrir dans les représentations des élèves ce

6. Jacques David et Harmony Gonsalves 2007. 
qu'E. Prairat désigne comme le «téléscopage axiologique » entre l'école et le monde qui l'entoure:

D'un côté, une société qui fait l'apologie de l'immédiateté, du zapping [...]; de l'autre une école qui en appelle au long terme, au plaisir différé, à l'abnégation et à une certaine gratuité de l'effort. Conflit de valeurs entre l'école et la société, qui fait de l'école un lieu exigeant, trop exigeant pour certains. (Prairat 2002 : 35.)

\section{Pour conclure}

Une recherche descriptive n'a d'autre ambition que celle de mettre à plat et de rendre mieux intelligible son objet, ici l'objet diffus dans tous les écrits des adolescents que sont leurs connaissances orthographiques. On espère contribuer à mieux caractériser « la baisse de l'orthographe », certes ressentie et invoquée dans le corps social comme problématique, mais commentée sans repères, et, à mon sens, de manière souvent aristocratique, entre désinvolture ou indignation, notamment par ceux pour qui l'orthographe n'est pas un obstacle. Les résultats dont on a fait état peuvent contribuer à nourrir positivement un débat récurrent, trop souvent mis en cause dans ses prémisses mêmes avant d'être mené à bien, celui des missions que la société confie à l'école en matière de savoirs exigibles de tous, ce fameux socle de connaissances, «impératif de culture commune, selon François Dubet, choix de justice fondamental car il préserve les plus faibles d'une dégradation de leur situation » (2004: 70). L'orthographe en fait-elle partie?

\section{BIBLIOGRAPHIE}

Blanche-Benveniste C. \& Chervel A. (1969), L'orthographe, Paris, Maspéro.

CATACH N. (1978), l'orthographe, Paris, PUF, Que sais-je?

Chervel A. (2006), Histoire de l'enseignement du français $d u X V I I^{e}$ au XX $X^{e}$ siècle, Paris, Retz.

Chervel A. \& MANesse D. (1989) La Dictée, les Français et l'orthographe 1873-1987, Calmann-Levy.

DAVID J. \& GONSALVES H (2007), «L'écriture électronique, une menace pour la maîtrise de la langue? ", Le français aujourd'hui, n 156. 
DuBET F. (2004), L'école des chances, Paris, Seuil.

Elalouf M.-L. et al. (1998), « Les futurs enseignants et l'orthographe Représentations et formation ", Le français aujourd'hui, Des conflits en orthographe, $n^{\circ} 122$.

GAK V.-G. (1976, trad. fçse), L'orthographe du français, essai de description théorique et pratique, Paris, SELAV.

Le français aujourd'hui (2007), $\mathrm{n}^{\circ} 156$.

MANESSE D. (dir.) (2003), Le Français dans les classes difficiles, le collège entre langue et discours, Paris, INRP.

MANesse D. \& CoGis D. (2007), L'orthographe: à qui la faute?, Paris, ESF.

Prairat E (2002), Questions de discipline à l'école, Ramonville-saint-Agne, Éditions Erès.

WYNANTS B. (1997), «Orthographe française. Altérations et crispations » in De Munck J. \& Verhoeven M., Les mutations du rapport à la norme. Un changement dans la modernité, De Boeck Université, coll. Ouvertures sociologiques : 69-86. 